\title{
HELICAL MAPS FROM LCA-GROUPS INTO HILBERT SPACES
}

\author{
JØRGEN CHRISTIAN RIDDER EBBESEN
}

\begin{abstract}
.
In this paper we study helical maps from LCA-groups into Hilbert spaces. The well known spectral representations of helices and their covariance kernels will be generalized to helical maps from arbitrary LCA-groups. The LévyHinčin representation of negative definite functions on LCA-groups is finally deduced from our spectral theorems.
\end{abstract}

\section{Introduction.}

Helices in metric spaces were introduced and studied by I. J. Schönberg and J. von Neumann in [10]. Most of their paper was devoted to the study of helices in real Hilbert spaces. Independently A. N. Kolmogorov [5] studied helices in complex Hilbert spaces and stated the classical representations of helices and their covariance kernels.

The modern investigation of helices in Hilbert spaces was initated by $P$. Masani [7], [8], to which we refer for further historical notes and applications.

The main source of inspiration of this paper has been [11] in which A. M. Yaglom studied stationary and helical maps on $R^{d}$ by means of generalized random fields. Schwartz's generalization of Bochner's theorem to positive definite distributions played an important role in [11, Theorem 1]. In the study of stationary maps Bochner's theorem applies directly to the covariance function, a fact used below to generalize the classical representation theorems to arbitrary LCA-groups.

\section{The spectral representation of covariance kernels.}

Throughout this paper $G$ denotes a locally compact abelian group (in short: LCA-group) with dual group $\Gamma . H$ denotes a Hilbert space (over C). The notation used follows [2]. 
Definition 1.1. Let $X$ be a topological space. A continuous kernel $K: X \times X \rightarrow C$ is called positive definite if the matrix $\left(K\left(x_{i}, x_{j}\right)\right)$ is positive hermitian for all $x_{1}, \ldots, x_{n} \in X, n \in \mathrm{N}$.

For later reference we state the following important theorem essentially due to A. N. Kolmogorov, cf. [6].

Theorem 1.2. Let $X$ be a topological space and $K: X \times X \rightarrow \mathrm{C}$ a continuous kernel. A necessary and sufficient condition that $K$ be positive definite is the existence of a Hilbert space $H$ and a norm continuous map $x: X \rightarrow H$ such that

$$
K(s, t)=\left(x_{s} \mid x_{t}\right), \quad s, t \in X .
$$

We omit the proof. It is an easy consequence of the theorem of N. Aronszajn $[1$, p. 143].

Definition 1.3. A norm continuous map $x: G \rightarrow H$ is called helical if the scalar product

$$
\left(x_{t_{1}+t}-x_{t_{2}+t} \mid x_{t_{3}+t}-x_{t_{4}+t}\right), \quad t_{1}, \ldots, t_{4} \in G,
$$

is independent of $t \in G$, and stationary if $\left(x_{s} \mid x_{t}\right)$ is a function of $s-t$ only. If $x: G \rightarrow H$ is helical, the kernel $(s, t) \rightarrow\left(x_{s}-x_{0} \mid x_{t}-x_{0}\right)$ is called the covariance kernel of $x$, and the chordal subspace $S_{x}$ of $x$ is defined by

$$
S_{x}=\mathrm{cl} \operatorname{span}\left\{x_{s}-x_{t} \mid s, t \in G\right\} .
$$

If $x: G \rightarrow H$ is stationary, the function $t \rightarrow\left(x_{t} \mid x_{0}\right)$ is called the covariance function of $x$.

Stationary maps are of course helical. If $x: G \rightarrow H$ is helical and $r \in G$, then $x^{r}: G \rightarrow H$ defined by

$$
x_{t}^{r}=x_{t+r}-x_{t}, \quad t \in G,
$$

is stationary, and so is any linear combination of the $x^{r}$ s.

Helical maps are essentially uniquely determined by their covariance kernels, as the following proposition shows.

Proposition 1.4. Assume that $x: G \rightarrow H$ and $\tilde{x}: G \rightarrow \tilde{H}$ are helical maps with the same covariance kernel. There exists a uniquely determined Hilbert space isomorphism $\Phi: S_{x} \rightarrow S_{\tilde{x}}$ satisfying

$$
\Phi\left(x_{t}-x_{0}\right)=\tilde{x}_{t}-\tilde{x}_{0}, \quad t \in G .
$$

Proof. Trivial. 
For stationary maps we have the following simple representation of the covariance function as an immediate consequence of Bochner's theorem [2, Theorem 3.12].

Proposition 1.5. Let $x: G \rightarrow H$ be stationary. There exists a unique measure $\mu \in M_{b}^{+}(\Gamma)$ such that

$$
\left(x_{t} \mid x_{0}\right)=\int(t, \gamma) d \mu(\gamma), \quad t \in G .
$$

Proof. The covariance function of a stationary map is obviously continuous and positive definite.

Lemma 1.6. Let $x: G \rightarrow H$ be helical and let $r_{1}, \ldots, r_{n} \in G$ be given. There exist $n^{2}$ uniquely determined measures $u_{r_{3}, r_{k}} \in M_{b}(\Gamma)$ such that

$$
\left(x_{s}^{r_{j}} \mid x_{t}^{r_{k}}\right)=\int(s-t, \gamma) d \mu_{r_{j}, r_{k}}(\gamma), \quad j, k=1, \ldots, n .
$$

Furthermore

$$
\sum_{j, k=1}^{n} c_{j} \bar{c}_{k} \mu_{r_{r}, r_{k}} \in M_{b}^{+}(\Gamma) \quad \text { for any }\left(c_{1}, \ldots, c_{n}\right) \in \mathrm{C}^{n} .
$$

Proof. For any $c=\left(c_{1}, \ldots, c_{n}\right) \in \mathrm{C}^{n}$ we define the stationary map $c \cdot x: G \rightarrow H$ by

$$
(c \cdot x)_{t}=\sum_{j=1}^{n} c_{j} x_{t}^{r_{j}}, \quad t \in G .
$$

According to Proposition 1.5 we may choose $\mu_{c} \in M_{b}^{+}(\Gamma)$ so that

$$
\left((c \cdot x)_{s} \mid(c \cdot x)_{t}\right)=\int(s-t, \gamma) d \mu_{c}(\gamma), \quad s, t \in G .
$$

We now define

$$
\mu_{r_{j}, r_{k}}=\frac{1}{4} \sum_{v=0}^{3} i^{v} \mu_{e_{j}+i^{v} e_{k}}, \quad j, k=1, \ldots, n,
$$

where $e_{1}, \ldots, e_{n}$ is the canonical base of $\mathrm{C}^{n}$. Formula (1.1) is now obvious.

The uniqueness part follows from the fact that the Fourier transform is injective as a mapping from $M_{b}(\Gamma)$, cf. [2, Proposition 2.3].

The last assertion of the lemma follows from 


$$
\sum_{j, k=1}^{n} c_{j} \bar{c}_{k} \mu_{r_{j}, r_{k}}=\mu_{c} \in M_{b}^{+}(\Gamma)
$$

The proof of the first part of the theorem below is inspired by [11, (especially pp. 285-289)]. The second part is due to B. Fuglede (oral communication).

Theorem 1.7. Let $x: G \rightarrow H$ be helical. There exist a unique measure $\mu \in M^{+}(\Gamma \backslash\{0\})$ satisfying

$$
\int(1-\mathrm{re}(t, \gamma)) d \mu(\gamma)<\infty, \quad t \in G
$$

and a unique biadditive positive definite kernel $B: G \times G \rightarrow C$ such that

$$
\left(x_{s}-x_{0} \mid x_{t}-x_{0}\right)=\int(1-(s, \gamma))(1-\overline{(t, \gamma)}) d \mu(\gamma)+B(s, t), \quad s, t \in G .
$$

Conversely, if $\mu$ and $B$ have the properties stated above, there exist a Hilbert space $H$ and a helical map $x: G \rightarrow H$ such that (1.2) holds.

Proof. Let $x: G \rightarrow H$ be helical. For $r_{1}^{\prime}, r_{1}^{\prime \prime}, r_{2} \in G$ we have according to Lemma 1.6

$$
\begin{aligned}
& \int(s-t, \gamma) d \mu_{r_{1}^{\prime}+r_{1}^{\prime \prime}, r_{2}}(\gamma)=\left(x_{s+r_{1}^{\prime}+r_{1}^{\prime \prime}}-x_{s} \mid x_{t+r_{2}}-x_{t}\right) \\
& \quad=\left(x_{s+r_{1}^{\prime}+r_{1}^{\prime \prime}}-x_{s+r_{1}^{\prime}} \mid x_{t+r_{2}}-x_{t}\right)+\left(x_{s+r_{1}^{\prime}}-x_{s} \mid x_{t+r_{2}}-x_{t}\right) \\
& \quad=\int(s-t, \gamma)\left(r_{1}^{\prime}, \gamma\right) d \mu_{r_{1}^{\prime \prime}, r_{2}}(\gamma)+\int(s-t, \gamma) d \mu_{r_{1}^{\prime}, r_{2}}(\gamma), \quad s, t \in G .
\end{aligned}
$$

From the calculation above and the injectivity of the Fourier transform we have

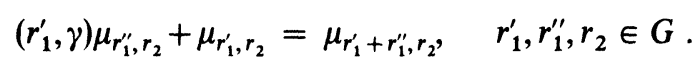

Interchanging $r_{1}^{\prime}$ and $r_{1}^{\prime \prime}$ in (1.3) leads to

$$
\left(1-\left(r_{1}^{\prime}, \gamma\right)\right) \mu_{r_{1}^{\prime \prime}, r_{2}}=\left(1-\left(r_{1}^{\prime \prime}, \gamma\right)\right) \mu_{r_{1}^{\prime}, r_{2}}, \quad r_{1}^{\prime}, r_{1}^{\prime \prime}, r_{2} \in G .
$$

From (1.4) and the obvious analogue

$$
\left(1-\overline{\left(r_{2}^{\prime}, \gamma\right)}\right) \mu_{r_{1}, r_{2}^{\prime \prime}}=\left(1-\left(\overline{r_{2}^{\prime \prime}, \gamma}\right)\right) \mu_{r_{1}, r_{2}^{\prime}}, \quad r_{1}, r_{2}^{\prime}, r_{2}^{\prime \prime} \in G,
$$

we obtain

$$
\left.\left(1-\left(r_{1}^{\prime}, \gamma\right)\right)\left(1-\left(\overline{r_{2}^{\prime}, \gamma}\right)\right) \mu_{r_{1}^{\prime \prime}, r_{2}^{\prime \prime}}=\left(1-\left(r_{1}^{\prime \prime}, \gamma\right)\right)\left(1-\overline{\left(r_{2}^{\prime \prime}, \gamma\right.}\right)\right) \mu_{r_{1}^{\prime}, r_{2}^{\prime}}
$$


for all $r_{1}^{\prime}, r_{1}^{\prime \prime}, r_{2}^{\prime}, r_{2}^{\prime \prime} \in G$.

Define $O_{r} \subset \Gamma \backslash\{0\}$ by

$$
O_{r}=\{\gamma \in \Gamma \mid(r, \gamma) \neq 1\}, \quad r \in G .
$$

We obviously have $\Gamma \backslash\{0\}=\cup_{r \in G} O_{r}$. On each $O_{r}$ we have the positive Radon measure $|1-(r, \gamma)|^{-2} \mu_{r, r}$. According to (1.6) the measures $\left|1-\left(r_{1}, \gamma\right)\right|^{-2} \mu_{r_{1}, r_{1}}$ and $\left|1-\left(r_{2}, \gamma\right)\right|^{-2} \mu_{r_{2}, r_{2}}$ agree on $O_{r_{1}} \cap O_{r_{2}}$. The measures $|1-(r, \gamma)|^{-2} \mu_{r, r}, r \in G$ can therefore be put together into a measure $\mu \in M^{+}(\Gamma \backslash\{0\})$ such that

$$
\mu_{r_{1}, r_{2} \mid \Gamma \backslash\{0\}}=\left(1-\left(r_{1}, \gamma\right)\right)\left(1-\overline{\left(r_{2}, \gamma\right)}\right) \mu, \quad r_{1}, r_{2} \in G .
$$

Inserting this in Lemma 1.6 gives

$$
\left(x_{s}^{r_{1}} \mid x_{t}^{r_{2}}\right)=\int(s-t, \gamma)\left(1-\left(r_{1}, \gamma\right)\right)\left(1-\left(\overline{r_{2}, \gamma}\right)\right) d \mu(\gamma)+\mu_{r_{1}, r_{2}}(\{0\})
$$

for all $s, t, r_{1}, r_{2} \in G$. If we put $s=t=0$ in (1.8) we obtain (1.2) with $B(s, t)$ $=\mu_{s, t}(\{0\}), s, t \in G$.

We still have to prove that $B$ has the properties stated in the theorem. The continuity of $B$ is obtained by proving that the integral in (1.2) is continuous, as the continuity of the left hand side is evident. Assume that the measure $\mu \in M^{+}(\Gamma \backslash\{0\})$ satisfies

$$
\int|1-(t, \gamma)|^{2} d \mu(\gamma)<\infty, \quad t \in G
$$

Define the map $y: G \rightarrow L^{2}(\mu)$ by

$$
y_{t}(\gamma)=1-(t, \gamma), \quad \gamma \in \Gamma \backslash\{0\}, t \in G .
$$

Then

$$
\begin{aligned}
\left\|y_{t}-y_{t_{0}}\right\|^{2} & =\int\left|1-\left(t-t_{0}, \gamma\right)\right|^{2} d \mu(\gamma) \\
& =\frac{1}{2} \int\left|1-\left(t-t_{0}, \gamma\right)\right|^{2} d(\mu+\check{\mu})(\gamma) \quad \text { for all } t_{0}, t \in G .
\end{aligned}
$$

According to [4, p. 528], $y: G \rightarrow L^{2}(\mu)$ is continuous (at $t_{0} \in G$ ). As

$$
\int(1-(s, \gamma))(1-\overline{(t, \gamma)}) d \mu(\gamma)=\left(y_{s} \mid y_{t}\right), \quad s, t \in G,
$$

the continuity assertion is proved.

The positive definiteness of $B$ follows from

$$
\sum_{j, k=1}^{n} c_{j} \bar{c}_{k} B\left(t_{j}, t_{k}\right)=\left(\sum_{j, k=1}^{n} c_{j} \bar{c}_{k} \mu_{t_{j}, t_{k}}\right)(\{0\}) \geqq 0
$$


for all $c_{1}, \ldots, c_{n} \in \mathrm{C}, n \in \mathrm{N}$, cf. Lemma 1.6.

The additivity of $B$ in the first variable follows from (1.3). The additivity in the second variable follows likewise. This completes the existence proof.

If (1.2) holds for some $\mu$ and $B$, we easily obtain

$$
\begin{aligned}
\left(x_{s}^{r_{1}} \mid x_{t}^{r_{2}}\right) & \left.=\int(s-t, \gamma)\left(1-\left(r_{1}, \gamma\right)\right)\left(1-\overline{\left(r_{2}, \gamma\right.}\right)\right) d \mu(\gamma)+B\left(r_{1}, r_{2}\right) \\
& =\int(s-t, \gamma) d v_{r_{1}, r_{2}}, \quad r_{1}, r_{2} \in G
\end{aligned}
$$

where the measure $v_{r_{1}, r_{2}} \in M_{b}(\Gamma)$ is defined by $v_{r_{1}, r_{2}}(\{0\})=B\left(r_{1}, r_{2}\right)$ and

$$
v_{r_{1}, r_{2} \mid \Gamma \backslash\{0\}}=\left(1-\left(r_{1}, \gamma\right)\right)\left(1-\left(\overline{r_{2}, \gamma}\right)\right) \mu .
$$

According to Lemma 1.6, $v_{r_{1}, r_{2}}$ is unique, hence the uniqueness of $B$ immediately follows. The uniqueness of $\mu$ also follows, as $\mu$ may be reconstructed from the measures $|1-(r, \gamma)|^{2} \mu, r \in G$.

Assume finally that $\mu$ and $B$ have the properties stated in the theorem. The map $y: G \rightarrow L^{2}(\mu)$ defined by (1.9) is obviously helical. According to Theorem 1.2 we may choose a Hilbert space $H$ and a norm continuous map $z: G \rightarrow H$, so that $B(s, t)=\left(z_{s} \mid z_{t}\right), s, t \in G$. Using the biadditivity of $B$ it is easily shown that $t \rightarrow z_{t}$ is helical. We conclude that $x=(y, z): G \rightarrow L^{2}(\mu) \oplus H$ is helical and that (1.2) holds.

For a positive Radon measure $\mu$ on $\mathbf{R}^{d} \backslash\{0\}$ the condition

$$
\int\left(1-\mathrm{re} e^{i(x \mid y)}\right) d \mu(y)<\infty, \quad x \in \mathbf{R}^{d}
$$

is easily seen to be satisfied if and only if

$$
\int \frac{\|y\|^{2}}{1+\|y\|^{2}} d \mu(y)<\infty .
$$

We therefore have the following result of A. M. Yaglom [11, p. 285, Theorem 6; p. 289, Remark 3] as a particular case of Theorem 1.7:

Corollary 1.8. Let $x: R^{d} \rightarrow H$ be helical. There exist a unique measure $\mu \in M_{b}^{+}\left(R^{d} \backslash\{0\}\right)$ and a unique positive hermitian $d \times d$ matrix $A$ of complex numbers, such that

$$
\left(x_{s}-x_{0} \mid x_{t}-x_{0}\right)=\int\left(1-e^{i(s \mid y)}\right)\left(1-e^{-i(t \mid y)}\right) \frac{1+\|y\|^{2}}{\|y\|^{2}} d \mu(y)+(A s \mid t)
$$

for all $s, t \in \mathbf{R}^{d}$. 


\section{The spectral representation of stationary and helical maps.}

In this section stationary and helical maps are represented in terms of certain vector integrals. The vector integration involved is outlined below. For a fuller account of the theory of CAOS-measures we refer to [9] and references therein. In the sequel $X$ denotes a locally compact Hausdorff space, $\boldsymbol{B}$ the Borel algebra on $X$ and $\boldsymbol{B}^{*}=\{B \in \boldsymbol{B} \mid \mathrm{cl} B$ is compact $\}$.

Definition 2.1. A map $\varrho: \boldsymbol{B}^{*} \rightarrow H$, which satisfies

$$
\begin{aligned}
B_{1} \cap B_{2}=\varnothing & \Rightarrow \varrho\left(B_{1}\right) \perp \varrho\left(B_{2}\right), \quad B_{1}, B_{2} \in B^{*}, \\
& \varrho\left(\cup B_{n}\right)=\sum \varrho\left(B_{n}\right),
\end{aligned}
$$

whenever $\left(B_{n}\right)$ is a sequence of disjoint sets from $\boldsymbol{B}^{*}$ with $\cup B_{n} \in \boldsymbol{B}^{*}$, and

$$
\forall \varepsilon>0 \forall B \in B^{*} \exists K \subset B, K \text { compact: }\|\varrho(B)-\varrho(K)\|<\varepsilon,
$$

is called a CAOS-measure (countably additive orthogonally scattered).

REMARK 2.2. If $\varrho$ is a CAOS-measure on $X$, it is easily shown that there exists a unique positive Radon measure $\mu_{\varrho}$ on $X$ such that $\mu_{\varrho}(B)=\|\varrho(B)\|^{2}, B \in B^{*}$. We have

$$
\left(\varrho\left(B_{1}\right) \mid \varrho\left(B_{2}\right)\right)=\mu_{\varrho}\left(B_{1} \cap B_{2}\right), \quad B_{1}, B_{2} \in B^{*} .
$$

Definition 2.3. Let $\varrho$ be a CAOS-measure on $X$. The subspace $S_{e}$ generated by $\varrho$ is defined by

$$
S_{Q}=\operatorname{cl} \operatorname{span}\left\{\varrho(B) \mid B \in B^{*}\right\} .
$$

Let $S=\operatorname{span}\left\{1_{B} \mid B \in B^{*}\right\} \subset L^{2}\left(\mu_{e}\right)$. For $f=\sum_{i=1}^{n} c_{i} 1_{B_{i}}, c_{i} \in \mathrm{C}, B_{i} \in B^{*}, n \in \mathbf{N}$ we define

$$
\psi(f)=\sum_{i=1}^{n} c_{i} \varrho\left(B_{i}\right) .
$$

The map $\psi: S \rightarrow S_{e}$ thus defined is obviously isometric. It has a unique extension to an isometry $\tilde{\psi}: \operatorname{cl} S=L^{2}\left(\mu_{e}\right) \rightarrow S_{e}$. We define

$$
\int f d \varrho=\tilde{\psi}(f), \quad f \in L^{2}\left(\mu_{e}\right) .
$$

As an immediate consequence of the definition we have Proposition 2.4. Let $f, g \in L^{2}\left(\mu_{e}\right), \lambda, v \in \mathrm{C}$. Then 


$$
\begin{gathered}
\int(\lambda f+\mu g) d \varrho=\lambda \int f d \varrho+\mu \int g d \varrho . \\
\left(\int f d \varrho \mid \int g d \varrho\right)=\int f \bar{g} d \mu_{\varrho} .
\end{gathered}
$$

According to $(2.5)$ the map $\psi: L^{2}\left(\mu_{Q}\right) \rightarrow S_{Q}$ is surjective, hence a Hilbert space isomorphism. We have the following result in the opposite direction, denoting by $\mu$ a given positive Radon measure on $X$.

Proposition 2.5. Let $\psi: L^{2}(\mu) \rightarrow U$ be a Hilbert space isomorphism onto a closed subspace $U$ of $H$. There exists a unique CAOS-measure $\varrho$ on $X$ such that

$$
\psi(f)=\int f d \varrho, \quad f \in L^{2}(\mu) .
$$

Furthermore $\mu_{\mathrm{e}}=\mu$ and $S_{\mathrm{e}}=U$.

Proof. The uniqueness is evident as the only possible choice of $\varrho$ is

$$
\varrho(B)=\psi\left(1_{B}\right), \quad B \in B^{*} .
$$

We now use (2.8) as the definition of $\varrho$, and the proposition follows easily.

Lemma 2.6. Let $\mu \in M_{b}^{+}(\Gamma)$. Then $\operatorname{cl} \operatorname{span}\{\gamma \rightarrow(t, \gamma) \mid t \in G\}=L^{2}(\mu)$.

Proof. Let $f \in L^{2}(\mu)$ and assume that

$$
0=\int f(\gamma) \overline{(t, \gamma)} d \mu(\gamma)=(f \mu) \hat{(t),} \quad t \in G .
$$

From the injectivity of the Fourier transform we conclude that $f=0$ in $L^{2}(\mu)$.

THEOREM 2.7. The map $x: G \rightarrow H$ is stationary if and only if there exists $a$ CAOS-measure $\varrho$ on $\Gamma$ with bounded $\mu_{\mathrm{e}}$ such that

$$
x_{t}=\int(t, \gamma) d \varrho(\gamma), \quad t \in G .
$$

In the affirmative case $\varrho$ is unique.

Proof. Let $\varrho$ be a CAOS-measure on $\Gamma$ with $\mu_{\ell}(\Gamma)<\infty$. Define $x: G \rightarrow H$ by (2.9). To $\varepsilon>0$ we may choose $K \subset \Gamma$ compact, so that $\mu_{\ell}(\Gamma \backslash K)<\varepsilon$. As $K$ is compact we may choose a neighbourhood $U$ of $t_{0} \in G$ so that

$$
\left|(t, \gamma)-\left(t_{0}, \gamma\right)\right|^{2}<\varepsilon, \quad \gamma \in K, t \in U \text {. }
$$


Hence by (2.6) and (2.7)

$$
\begin{aligned}
\left\|x_{t}-x_{t_{0}}\right\|^{2} & =\int\left|(t, \gamma)-\left(t_{0}, \gamma\right)\right|^{2} d \mu_{e}(\gamma) \leqq \varepsilon\left(\mu_{e}(K)+4\right) \\
& \leqq \varepsilon\left(\mu_{e}(\Gamma)+4\right), \quad t \in U .
\end{aligned}
$$

As $\varepsilon>0$ and $t_{0} \in G$ were arbitrary the continuity of $t \rightarrow x_{t}$ is established, and it follows by appeal to (2.7) that $t \rightarrow x_{t}$ is stationary.

Assume conversely that $x: G \rightarrow H$ is stationary and let $\left(x_{t} \mid x_{0}\right)=\int(t, \gamma) d \mu(\gamma)$, $t \in G$ be the representation of the covariance function, cf. Proposition 1.5. Define the isometry $\psi$ from the subspace $\operatorname{span}\left\{\hat{\varepsilon}_{t} \mid t \in G\right\}$ of $L^{2}(\mu)$ into $H$ by

$$
\psi\left(\sum_{i=1}^{n} c_{i} \hat{\varepsilon}_{-t_{i}}\right)=\sum_{i=1}^{n} c_{i} x_{t_{i}}, \quad c_{i} \in \mathrm{C}, t_{i} \in G, n \in \mathrm{N} .
$$

According to Lemma 2.6, $\psi$ may be extended to an isometry $\Psi: L^{2}(\mu) \rightarrow H$. According to Proposition 2.5 we may choose a CAOS-measure $\varrho$ on $\Gamma$ such that

$$
\widetilde{\psi}(f)=\int f d \varrho, \quad f \in L^{2}(\mu) .
$$

For $f=\hat{\varepsilon}_{-t}(2.10)$ leads to (2.9).

If (2.9) holds for some CAOS-measure $\varrho$ on $\Gamma$ with bounded $\mu_{\boldsymbol{e}}$, it follows that $\left(x_{t} \mid x_{0}\right)=\int(t, \gamma) d \mu_{e}(\gamma), t \in G$. This means that $\mu_{e}$ is the unique measure in the representation of the covariance function. The uniqueness of $\varrho$ now follows from Lemma 2.6.

Lemma 2.8. Let $\mu$ be a positive Radon measure on $\Gamma \backslash\{0\}$ which satisfies

$$
\int(1-\mathrm{re}(t, \gamma)) d \mu(\gamma)<\infty, \quad t \in \cdot G .
$$

Then cl span $\left\{\omega_{t} \mid t \in G\right\}=L^{2}(\mu)$, where $\omega_{t}: \Gamma \backslash\{0\} \rightarrow C$ is defined by $\omega_{t}(\gamma)=$ $1-(t, \gamma), \gamma \in \Gamma \backslash\{0\}$.

Proof. Let $f \in L^{2}(\mu)$ and assume that $\int \omega_{t} \bar{f} d \mu=0, t \in G$. As $\omega_{s} \bar{\omega}_{t}=\omega_{s}+\omega_{-t}$ $-\omega_{s-t}$, we have

$$
\int \omega_{s} \bar{\omega}_{t} \bar{f} d \mu=0, \quad s, t \in G .
$$

Interchanging $s$ and $t$ we obtain

$$
\int \omega_{s} \bar{\omega}_{t} f d \mu=0, \quad s, t \in G .
$$


Adding and subtracting these equations give

$$
\int \omega_{s} \bar{\omega}_{t} \operatorname{re} f d \mu=\int \omega_{s} \bar{\omega}_{t} \operatorname{im} f d \mu=0, \quad s, t \in G .
$$

From the uniqueness part of Theorem 1.7 we conclude re $f \cdot \mu=\operatorname{im} f \cdot \mu=0$, hence $f=0$ in $L^{2}(\mu)$.

The following complement to Theorem 1.7 is due to B. Fuglede (oral communication).

THEOREM 2.9. Let $x: G \rightarrow H$ be helical. There exist a unique CAOS-measure $\varrho$ on $\Gamma \backslash\{0\}$ with $\int(1-\mathrm{re}(t, \gamma)) d \mu_{e}<\infty, t \in G$ and a unique continuous additive map l: $G \rightarrow H$ satisfying $\int(1-(t, \gamma)) d \varrho(\gamma) \perp l(s)$ for all $s, t \in G$ such that

$$
x_{t}-x_{0}=\int(1-(t, \gamma)) d \varrho(\gamma)+l(t), \quad t \in G .
$$

Conversely, if $\varrho$ and $l$ have the properties stated above, there exist a Hilbert space $H$ and a helical map $x: G \rightarrow H$ so that (2.11) holds.

Proof. If

$$
\left(y_{s}-y_{0} \mid y_{t}-y_{0}\right)=\int(1-(s, \gamma))(1-\overline{(t, \gamma)}) d \mu(\gamma), \quad s, t \in G,
$$

for some $\mu \in M^{+}(\Gamma \backslash\{0\})$ such that $\int(1-\operatorname{re}(t, \gamma)) d \mu(\gamma)<\infty, t \in G$, then a CAOS-measure $\varrho$ on $\Gamma \backslash\{0\}$ such that

$$
y_{t}-y_{0}=\int(1-(t, \gamma)) d \varrho(\gamma), \quad t \in G,
$$

is obtained by a construction analogous to the construction in Theorem 2.7, using Lemma 2.8. If

$$
\left(z_{s} \mid z_{t}\right)=B(s, t), \quad s, t \in G,
$$

where $B$ is a biadditive positive definite kernel, it easily follows that $t \rightarrow z_{t}$ is a continuous additive map. The representation is now obvious for helical maps of the form

$$
\tilde{x}=(y, z): G \rightarrow H_{1} \oplus H_{2},
$$

where $y: G \rightarrow H_{1}$ satisfies (2.12) and $z: G \rightarrow H_{2}$ satisfies (2.13).

Let $x: G \rightarrow H$ be an arbitrary helical map. As noticed in the proof of Theorem 1.7, $x$ has its covariance kernel in common with a map $\tilde{x}$ of the above 
form. According to Proposition 1.4 we may write $x_{t}=\Phi \tilde{x}_{t}+a, t \in G$, where $\Phi: S_{\tilde{x}} \rightarrow S_{x}$ is a Hilbert space isomorphism and $a \in H$. From the representation of $\tilde{x}$

$$
\tilde{x}_{t}-\tilde{x}_{0}=\int(1-(t, \gamma)) d \varrho \tilde{\varrho}(\gamma)+\tau(t), \quad t \in G,
$$

with $\int(1-(t, \gamma)) d \tilde{\varrho}(\gamma) \perp \tau(s), \quad s, t \in G, \quad(2.11)$ follows with $\varrho=\Phi \circ \tilde{\varrho}, \quad l=\Phi \circ \tau$, and we obtain $\int(1-(t, \gamma)) d p(\gamma) \perp l(s)$ for all $s, t \in G$.

If (2.11) holds for some $\varrho$ and $l$ with the properties stated, we first note that $\mu_{\ell}$ is the unique measure in the representation (1.2) of the covariance kernel. The uniqueness of $\varrho$ then follows from Lemma 2.8, and the uniqueness of $l$ is now obvious.

The converse part is trivial.

\section{The Lévy-Hinčin representation of negative definite functions.}

In [8] P. Masani proved the classical Lévy-Hinčin representation of negative definite functions on $R^{d}$ by "helical" methods. In this section it is shown that these methods apply to arbitrary LCA-groups. The resulting formula is well known, cf. [3] and references therein. The basic difference between G. Forst's method and ours is found in the construction of the Lévy measure of a negative definite function. In [3] the Lévy measure is defined in terms of the associated convolution semigroup, whereas we obtain the Lévy measure from the associated positive definite kernel.

Lemma 3.1. Let $g$ be a Lévy function on $G \times \Gamma$ ([3, Definition 4]) and let $\mu$ be a positive Radon measure on $G \backslash\{0\}$ satisfying

$$
\int(1-\operatorname{re}(t, \gamma)) d \mu(t)<\infty, \quad \gamma \in \Gamma .
$$

The function $\psi: \Gamma \rightarrow \mathrm{C}$ defined by

$$
\left.\psi(\gamma)=\int(1-\overline{(t, \gamma})+i g(t, \gamma)\right) d \mu(t), \quad \gamma \in \Gamma
$$

is continuous and negative definite.

Proof. The assertion above is part of [3, Lemma 5].

THEOREM 3.2. Let $\mathrm{g}$ be a fixed Lévy function on $G \times \Gamma$. A function $\psi: \Gamma \rightarrow \mathrm{C}$ is continuous, negative definite with $\psi(0)=0$ if and only if it has the form 


$$
\left.\psi(\gamma)=i l(\gamma)+q(\gamma)+\int(1-\overline{(t, \gamma})+i g(t, \gamma)\right) d \mu(t), \quad \gamma \in \Gamma,
$$

where $l: \Gamma \rightarrow \mathrm{R}$ is continuous and additive, $q$ is a continuous, non-negative quadratic form ([2, Definition 7.18]), and $\mu \in M^{+}(G \backslash\{0\})$ satisfies $\int(1-\mathrm{re}(t, \gamma)) d \mu(t)<\infty, \gamma \in \Gamma$.

In the affirmative case $l, q, \mu$ are unique.

Proof. The "if"-part is obvious from Lemma 3.1. Assume conversely that $\psi: \Gamma \rightarrow \mathrm{C}$ is continuous, negative definite with $\psi(0)=0$. As the kernel $K_{\psi}$ defined by

$$
K_{\psi}\left(\gamma_{1}, \gamma_{2}\right)=\psi\left(\gamma_{1}\right)+\overline{\psi\left(\gamma_{2}\right)}-\psi\left(\gamma_{1}-\gamma_{2}\right), \quad \gamma_{1}, \gamma_{2} \in \Gamma
$$

is positive definite, we may choose a Hilbert space $H$ and a continuous map $x: \Gamma \rightarrow H$ so that

$$
K_{\psi}\left(\gamma_{1}, \gamma_{2}\right)=\left(x_{\gamma_{1}} \mid x_{\gamma_{2}}\right), \quad \gamma_{1}, \gamma_{2} \in \Gamma,
$$

cf. Theorem 1.2. From (3.2) it follows that $x: \Gamma \rightarrow H$ is helical. It also follows that $x_{0}=0$, so according to Theorem 1.7 we have

$$
K_{\psi}\left(\gamma_{1}, \gamma_{2}\right)=\int\left(1-\left(\overline{t, \gamma_{1}}\right)\right)\left(1-\left(t, \gamma_{2}\right)\right) \cdot d \mu(t)+B\left(\gamma_{1}, \gamma_{2}\right), \quad \gamma_{1}, \gamma_{2} \in \Gamma
$$

for some $\mu \in M^{+}(G \backslash\{0\})$ satisfying $\int(1-\operatorname{re}(t, \gamma)) d \mu(t)<\infty, \gamma \in \Gamma$ (note that $\mu$ in (3.3) corresponds to $\check{\mu}$ in (1.2)), and some biadditive positive definite kernel $B$. From the biadditivity of $B$ it follows that $B\left(-\gamma_{1},-\gamma_{2}\right)=B\left(\gamma_{1}, \gamma_{2}\right), \gamma_{1}, \gamma_{2} \in \Gamma$. From the definition (3.2) of $K_{\psi}$ we have $K_{\psi}\left(-\gamma_{1},-\gamma_{2}\right)=\overline{K_{\psi}\left(\gamma_{1}, \gamma_{2}\right)}, \gamma_{1}, \gamma_{2} \in \Gamma$. Using (3.3) we therefore obtain

$$
\begin{aligned}
B\left(\gamma_{1}, \gamma_{2}\right) & =B\left(-\gamma_{1},-\gamma_{2}\right)=K_{\psi}\left(-\gamma_{1},-\gamma_{2}\right)-\int\left(1-\left(t, \gamma_{1}\right)\right)\left(1-\overline{\left(t, \gamma_{2}\right)}\right) d \mu(t) \\
& =\overline{B\left(\gamma_{1}, \gamma_{2}\right)}, \quad \gamma_{1}, \gamma_{2} \in \Gamma,
\end{aligned}
$$

i.e. $B$ only takes real values. We now construct the continuous, negative definite function

$$
\psi_{x}(\gamma)=q(\gamma)+\int(1-\overline{(t, \gamma)}+i g(t, \gamma)) d \mu(t), \quad \gamma \in \Gamma,
$$

where $q(\gamma)=\frac{1}{2} B(\gamma, \gamma), \gamma \in \Gamma$, obviously is a continuous, non-negative quadratic form. Let $K_{\psi_{x}}: \Gamma \times \Gamma \rightarrow \mathrm{C}$ be the positive definite kernel corresponding to $\psi_{x}$, i.e.

$$
K_{\psi_{x}}\left(\gamma_{1}, \gamma_{2}\right)=\psi_{x}\left(\gamma_{1}\right)+\overline{\psi_{x}\left(\gamma_{2}\right)}-\psi_{x}\left(\gamma_{1}-\gamma_{2}\right), \quad \gamma_{1}, \gamma_{2} \in \Gamma
$$


Using that $B$ is real valued we obtain $K_{\psi}=K_{\psi_{x}}$, from which it easily follows that $\psi-\psi_{x}=i l$, where $l: \Gamma \rightarrow \mathrm{R}$ is continuous and additive. We therefore have

$$
\begin{aligned}
\psi(\gamma) & =i l(\gamma)+\psi_{x}(\gamma) \\
& \left.=i l(\gamma)+q(\gamma)+\int(1-\overline{(t, \gamma})+i g(t, \gamma)\right) d \mu(t), \quad \gamma \in \Gamma,
\end{aligned}
$$

which proves the "only if"-part.

If (3.1) holds for some $l, q$ and $\mu$ with the properties stated, then

$$
K_{\psi}\left(\gamma_{1}, \gamma_{2}\right) \cdot \int\left(1-\left(t, \gamma_{1}\right)\right)\left(1-\overline{\left(t, \gamma_{2}\right)}\right) d \check{\mu}(t)+B\left(\gamma_{1}, \gamma_{2}\right), \quad \gamma_{1}, \gamma_{2} \in \Gamma,
$$

where $B\left(\gamma_{1}, \gamma_{2}\right)=q\left(\gamma_{1}\right)+q\left(\gamma_{2}\right)-q\left(\gamma_{1}-\gamma_{2}\right), \gamma_{1}, \gamma_{2} \in \Gamma$, is a biadditive positive definite kernel, cf. [2, p. 47]. According to Theorem 1.7, $K_{\psi}$ is therefore the covariance kernel of some helical map $x: \Gamma \rightarrow H, H$ Hilbert space. From the uniqueness part of the same theorem the uniqueness of $\mu$ and $q$ immediately follows (as $q(0)=0$ we necessarily have $q(\gamma)=\frac{1}{2} B(\gamma, \gamma), \gamma \in \Gamma$ ). The uniqueness of $l$ is now obvious.

Acknowledgement. The author is indebted to professor Bent Fuglede for inspiring conversations and for stimulating the work on this paper.

\section{REFERENCES}

1. N. Aronszajn, La théorie des noyeaux reproduisants et ses applications I, Proc. Cambridge Philos. Soc. 39 (1943), 133-153.

2. C. Berg and G. Forst, Potential theory on locally compact Abelian groups (Ergeb. Math. Grenzgeb. 87), Springer-Verlag, Berlin - Heidelberg - New York, 1975.

3. G. Forst, The Lévy-Hinčin representation of negative definite functions, $\mathrm{Z}$. Wahrsch. Verw. Gebiete 34 (1976), 313-318.

4. K. Harzallah, Sur une demonstration de la formule de Lévy-Hinčin, Ann. Inst. Fourier (Grenoble) 19 (1969), 527-532.

5. A. N. Kolmogorov, Kurven in Hilbertschen Raum, die gegenüber einer einparametrigen Gruppe von Bewegungen invariant sind, Dokl. Akad. Nauk SSSR 26 (1940), 6-9.

6. A. N. Kolmogorov, Stationary sequences in Hilbert space, Moscow Univ. Bull. Math. 2 (1941), $1-40$.

7. P. Masani, On helixes in Hilbert space I, Theory Probab. Appl. 17 (1972), 1-19.

8. P. Masani, On infinitely decomposable probability distributions, and helical varieties in Hilbert space, in Multivariate Analysis III, (Proc. Dayton, Ohio, 1972), ed. P. R. Krisnaiah, pp. 209 223. Academic Press, New York, 1973. 
9. P. Masani, Orthogonally scattered measures, Adv. in Math. 2 (1968), 61-117.

10. I. J. Schönberg and J. von Neumann, Fourier integrals and metric geometry, Trans. Amer. Math. Soc. 50 (1941), 226-251.

11. A. M. Yaglom, Some classes of random fileds in n-dimensional space, related to stationary random processes, Theory Probab. Appl. 2 (1957), 273-320.

AMAGERF ELLEDVEJ $9^{\text {IV }}$, TH

DK-2300 KØBENHAVN S

DENMARK 